\title{
Correction
}

\section{Correction: Wen et al., Dynamic Range Adaptation to Sound Level Statistics in the Auditory Nerve}

In the article "Dynamic Range Adaptation to Sound Level Statistics in the Auditory Nerve" by Bo Wen, Grace I. Wang, Isabel Dean, and Bertrand Delgutte, which appeared on pages 13797-13808 of the November 4, 2009 issue, the sound pressure levels of all broadband noise (BBN) stimuli were reported incorrectly due to a programming error. Specifically, the actual sound levels of BBN stimuli were 6 $\mathrm{dB}$ lower than the reported values. The error did not affect levels of pure tone stimuli, which were correctly reported.

As a result, $6 \mathrm{~dB}$ should be subtracted from all the axis labels referring to BBN stimulus levels in Figures 4, 7, 9A, and $9 C$, including $\mathrm{HPR}$ mean levels and the level-dependent metrics $L_{\mathrm{th}}, L_{50}, L_{\mathrm{sat}}, L_{\delta^{\prime} \max }$, and $L_{\mathrm{FImax}}$. Two other figure panels, Figures $8 B$ and $9 B$, cannot be corrected by simply subtracting $6 \mathrm{~dB}$ from the axes because they include both data that are affected by the error and data that are not. We provide updates for these two panels below.

Importantly, the main characteristics of dynamic range adaptation, i.e., the amounts of shifts in rate-level functions, and the rates of growth of adaptation (i.e., $L_{\text {th }}$ slopes, $L_{50}$ slopes, and $L_{\text {sat }}$ slopes) were correctly reported because these difference metrics are not affected by a constant level offset. For this reason, the error does not alter any of our conclusions about dynamic range adaptation in the auditory nerve and the comparison with adaptation in the inferior colliculus.

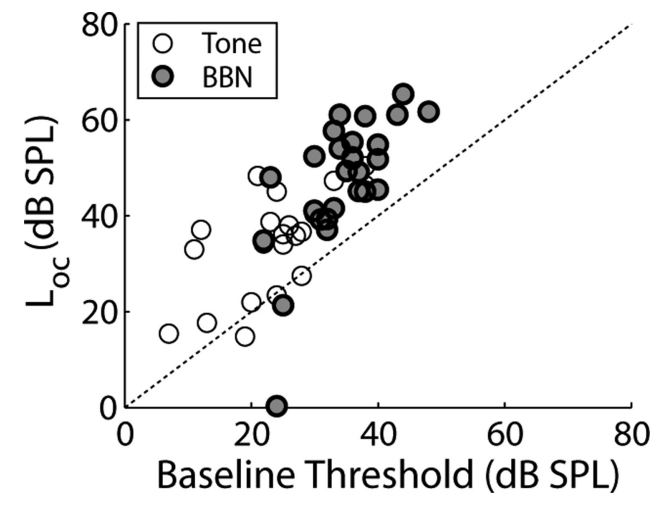

Figure $8 B$.

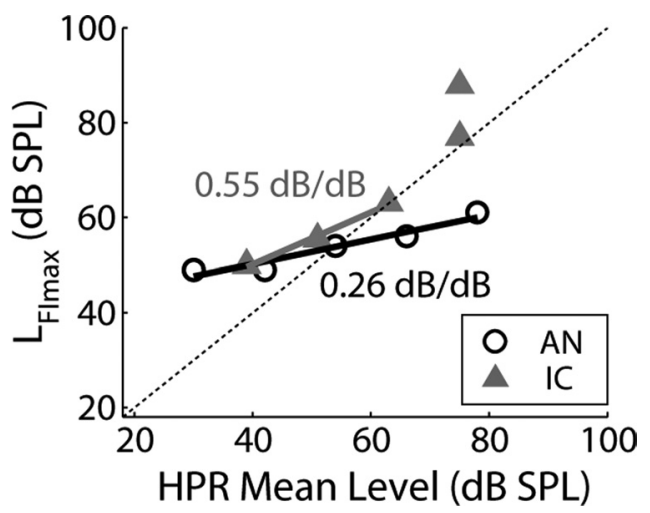

Figure $9 B$.

DOI: 10.1523/JNEUROSCI.2164-12.2012 